\title{
Effects of session and intrasession repetition on individual power law exponents
}

\author{
WILLIAM E. DAWSON and STEVEN P. WATERMAN \\ University of Notre Dame, Notre Dame, Indiana 46556
}

\begin{abstract}
Number of presentations of the stimulus series was manipulated to choose between response bias and transitory memory theories of individual differences in power function exponents. Four groups of 15 subjects gave magnitude estimations of circle areas in two sessions separated by 1 week. The number of first session presentations for a group was one, two, four, or eight; all received three second session repetitions. Interrepetition correlations of exponents for individuals for adjacent repetitions, within and across sessions, were significant, indicating reliable subject differences. Correlations remained stable over presentations and were similar across groups, suggesting little effect due to repetition. Correlations between sessions did decrease, as the memory hypothesis would predict, but not significantly. Results were interpreted as favorable to the response bias hypothesis but a rapid learning explanation could not be ruled out.
\end{abstract}

As the literature on Stevens' power law and its applications steadily grows (Stevens, 1975), the problems with his direct scaling methods become of more glaring importance. One problem has been the large intersubject variation in exponents (Jones \& Marcus, 1961). Even Stevens (1975) has noted that this variability seems unduly large given the levels of variability in sensory processes. It has led Luce (1972) to suggest psychophysical measurement may be fundamentally different from physical measurement and, further, that individual differences may not permit a unit of sensory magnitude independent of the individual.

Two noteworthy efforts have been made to deal with this important problem. Stevens (1975) hypothesized that intersubject differences are mainly due to the regression effect and that a compensatory balance for this effect would greatly reduce the variation. However, recent research (Dawson \& Mirando, 1975; Wanschura \& Dawson, 1974) shows a sizeable variability in exponents remains after such a balance. The second effort, that of Teghtsoonian and Teghtsoonian (1971), involved noting conditions where individuals appear to contribute little systematic variance to the scaling results. They found interrepetition correlations of exponents, a measure of persisting individual differences used by Rule (1966), dropped to nonsignificance for intervals as short as $24 \mathrm{~h}$ if the observers were shown the stimuli only once per session. Under these conditions exponent difference was a random effect suggesting one need not accept Luce's contention concerning units of sensory magnitude. However, Engeland and Dawson (1974), using a somewhat different procedure than Teghtsoonian and Teghtsoonian (1971), failed to find

Requests for reprints should be sent to William E. Dawson, Department of Psychology, University of Notre Dame, Notre Dame, Indiana 46556. nonsignificant correlations for both magnitude estimation and cross-modality matching with an intersession interval of 1 week. The study differed in that one of its experiments employed cross-modality matching; the Teghtsoonian experiments used magnitude estimation. It also differed in that its magnitude estimation experiment used two presentations of each stimulus per session, a procedure contrary to the recommendation of their earlier paper.

The present study examined the effect of a week interval with only one presentation per stimulus in magnitude estimation. Its main goal, however, was to delineate the nature and source of differences in exponents. Are they due to a transitory memory effect, as implied by Teghtsoonian and Teghtsoonian, or are they the result of an ever-present response bias, as suggested by Rule? If a stable response bias is the source, then the differences should be unaffected by repeated presentations of the stimuli both within and across sessions. If the source is some memory effect, then supposedly it will be a function of the number of presentations per stimulus, as suggested by Teghtsoonian and Teghtsoonian. The experiment conducted varied the number of presentations in the first session for different groups of subjects; the second session was the same for all subjects and took place 1 week later.

\section{METHOD}

\section{Subjects}

Sixty undergraduates, five female, served as subjects. All were given credit in introductory psychology courses for their help. When they volunteered, they were told about the second session but nothing concerning their task at that time.

\section{Apparatus}

Several sets of eight circles were drawn in black ink, with one circle each to an $81 / 2 \times 11$ in. $(21.6 \times 27.9 \mathrm{~cm})$ sheet of white paper. Circle areas varied from 22.35 to $22,350 \mathrm{~mm}^{2}$ in a 
geometric progression. Each sheet of paper was fastened into a manila filing folder.

\section{Procedure}

Subjects were divided into four equal groups that received one, two, four, or eight presentations of the eight-circle series in the first session. All groups were given three presentations of the series in the second session. Subjects, one at a time, gave estimations of the apparent area of the circles. The first stimulus in each session was selected randomly from the four midrange circles and the subject instructed to give any number that seemed to him appropriate. Subsequent stimuli were to be judged in a proportional manner and they occurred in different irregular orders for each person. Subjects were told explicitly to judge apparent area and not to judge the actual physical areas (cf. Teghtsoonian, 1965), some linear aspect such as radius, or on the basis of their geometrical knowledge of circles. Subjects were randomly assigned to the four conditions in blocks of four to insure that subjects from the various groups were distributed evenly over time and other nonmanipulated conditions.

\section{RESULTS}

\section{Repetition Effects on Exponents}

For each group, geometric means were calculated for the judgments of every circle. Using a least-squares criterion, power functions were fitted to these valuesone for each group.They fit the data very well in all four cases. For the one-, two-, four-, and eight-repetition groups, respectively, means and standard deviations of the exponents were $.66 \pm .16, .61 \pm .18, .54 \pm .11$, and $.63 \pm .14$. Although the four-repetition exponent was lower. than the others, it was not significantly so, nor were any of the remaining comparisons statistically significant. Magnitudes of the exponents were close to the .70 reported by Stevens (1961) for visual area.

To assess effects of repetition on exponents, group exponents were found for each series presentation. Analyses of variance for each group showed repetitions gave an increase in exponent for all but the tworepetition group; the following Fs were obtained: $\mathrm{F}(3,42)=7.37, \mathrm{p}<.01 ; \mathrm{F}(4,56)=.486$, n.s.; $\mathrm{F}(6,84)$ $=3.97, \mathrm{p}<.01 ;$ and $\mathrm{F}(10,140)=2.27, \mathrm{p}<.05$. Although these effects were significant, in no case was the exponent for the first repetition of the first session statistically different from the comparable one of the second session.

A groups by repetitions analysis of variance was performed on the exponents for the second session data of the four groups to see if the number of repetitions in the first session had any effect here. An $F(3,56)=1.84$ indicated no differences in exponents among the groups but the repetitions effect, $F(2,112)$ $=8.56, \mathrm{p}<.01$, was significant. The interaction of Groups by Repetitions was not significant. Comparisons of the first presentation mean exponent with the means of second and third presentations found both of the latter to be significantly greater $(p<.01)$. Thus, exponents increased in the second session as they had during the first session, but the groups did not differ.

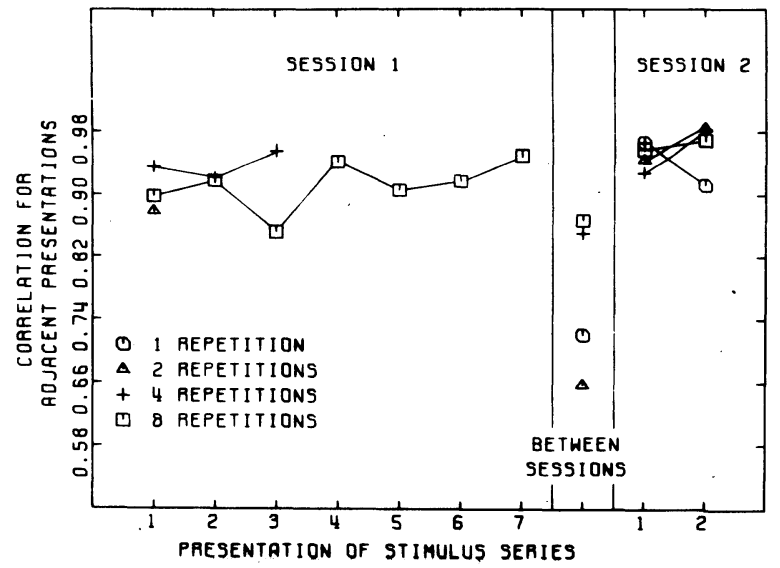

Figure 1. Product-moment correlations of individual exponents for adjacent presentations within and between the two sessions.

\section{Intersession Correlations}

Power function exponents were computed for each subject for each stimulus series presentation received. Product-moment correlations between exponents of the last presentation of the first session and first presentation of the second session were obtained for all groups. In order of number of first session repetitions, correlations were $.72, .66, .85$, and .87 (see Figure 1). All were significantly different from zero $(\mathrm{p}<.01)$. The trend is for an increase in correlation, expected if memory is being enhanced by repetition, but none of the differences among correlations was statistically significant. This fact suggests repetition has little to no effect in producing individual differences, at least after the second repetition. Rather, as the one-repetition group shows, a significant intersession correlation occurs with only one prior presentation, even with a week delay between presentations. Correlations start high and remain there, a finding supportive of the hypothesis of an ever-present response bias.

\section{Intrasession Correlations}

Figure 1 shows correlations between exponents for adjacent presentations for each of the groups. Thus, it includes intrasession correlations plus the four intersession correlations discussed above. All correlations were significantly different from zero at the .01 level. All first session values were in the neighborhood of .90 , even those for the first and second repetitions, suggesting subject differences are prominent from the beginning. The correlations remain at nearly the same level over additional repetitions. Although they increase slightly, as would be expected for a memory effect, they do not do so significantly. Hence, again, the memory effect, if it occurs, is mainly restricted to the first two presentations. Significant changes in correlations would be difficult to detect here, since correlations are so large to start with that a ceiling effect may mask changes. 
The two-repetition group shows the largest drop in correlation as it moves from the first session to the intersession coefficient, i.e., from .88 to .66. The two correlations, however, were not significantly different at the .05 level.

The effect of repetitions on the correlations of the second session also can be seen in Figure 1. Clearly, the groups differ little here; number of first session repetitions produces no statistically significant differences. Again, note that correlations are high from the start and a ceiling effect could be obscuring a memory effect here.

\section{DISCUSSION}

The transitory memory and response bias hypotheses predict different results. The obtained results can be assessed against these predictions. If response bias is held to be a constant and stable effect, then its impact on individual exponents should be stable. All interrepetition correlations, within or across sessions, would be of the same magnitude and significant from the outset. And so they are, according to the statistical analyses. Individual differences persist across presentations even while mean exponents of the groups show change. Thus, all subjects appear to change but their exponents remain in approximately the same relative order. At least two different sources of variance appear to be operative. Interestingly, exponents appear to increase in both sessions, after showing a decrease between sessions. The decrease yields statistically equivalent mean exponents for the first repetitions of the two sessions. One is reminded of the range effects discussed by Stevens (1975). The 1000:1 stimulus range is quite large for a visual area experiment and subjects may expand their range of numbers after learning just how large it is on the first presentations. The significant changes in exponents with repetitions were not expected because earlier studies have more often than not found invariant exponents (e.g., Wanschura \& Dawson, 1974).

The transitory memory hypothesis predicts increasing correlations for repeated presentations within a session. This effect should be detectable over the earliest repetitions, after which asymptotic performance might be reached. Further, the hypothesis predicts an intersession correlation (last repetition of Session 1 vs. first of Session 2) that is smaller than the preceding adjacent intrasession correlation because of forgutting. This effect could be overridden by overlearning if a large number of repetitions preceded the intersession interval, but it is apt to occur for a small number of them.
The statistical analyses support neither of these predictions. But if one looks only at the size of the rs in Figure 1, and not at whether they are significantly different, they do rise and subjects are more reliable within a session than between sessions. Both of these trends fit with the memory hypothesis. However, if a memory effect is the only factor involved, learning must take place rapidly, since even one repetition leads to evidence for individual differences. Further, it occurs with a week-long intersession delay and, hence, this study joins that of Engeland and Dawson (1974) in disagreement with Teghtsoonian and Teghtsoonian (1971). It is still fossible both hypotheses are correct, with both factors being effective, but the present study rules out the likelihood that individual differences are the result of a gradually developing memory effect. The existence of high and unchanging correlations, present from the beginning, is more supportive of the hypothesis of a personal response bias of some sort. The latter may be due to idiosyncratic ranges (Rule, 1966) or to differences in ratio criterion (Stevens, 1961).

\section{REFERENCES}

Dawson, W. E., \& Mirando, M. A. Sensory-modality opinion scales for individual subjects. Perception \& Psychophysics, 1975, 17, 596-600.

Engeland, W., \& Dawson, W. E. Individual differences in power functions for a 1-week intersession interval. Perception \& Psychophysics, 1974, 15, 349-352.

Jones, F. N., \& Marcus, J. J. The subject effect in judgments of subjective magnitude. Journal of Experimental Psychology, $1961,61,40-44$.

LUCE, R. D. What sort of measurement is psychophysical measurement? American Psychologist, 1972, 27, 96-106.

Rule, S. Subject differences in exponents of psychophysical power functions. Perceptual and Motor Skills, 1966, 23, 1125-1126.

STEvens, S. S. The psychophysics of sensory function. In W. A. Rosenblith (Ed.), Sensory communication. New York: Wiley, 1961.

Stevens, S. S. Psychophysics. New York: Wiley, 1975.

Teghtsoonian, M. The judgment of size. American Journal of Psychology, 1965, 78, 392-402.

Teghtsoonian, M., \& Teghtsoonian, R. How repeatable are Stevens's power law exponents for individual subjects? Perception \& Psychophysics, 1971, 10, 147-149.

Wanschura, R. G., \& Dawson, W. E. Regression effect and individual power functions over sessions. Journal of Experimental Psychology, 1974, 102, 806-812.

(Received for publication November 24, 1975.) 